\title{
Load curve smoothing strategy based on unified state model of different demand side resources
}

\author{
Mingshen WANG ${ }^{1}$, Yunfei MU ${ }^{1}$, , Tao JIANG ${ }^{2}$, Hongjie JIA $^{1}$, \\ Xue LI $^{2}$, Kai HOU ${ }^{1}$, Tong WANG ${ }^{3}$
}

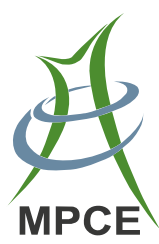

\begin{abstract}
Renewable energy based distributed generation (DG) has the potential to reach high penetration levels in the residential region. However, its integration at the demand side will cause rapid power fluctuations of the tieline in the residential region. The traditional generators are generally difficult to manage rapid power fluctuations due to their insufficient efficiency requirements and low responding speed. With an effective control strategy, the demand side resources (DSRs) including DGs, electric vehicles and thermostatically-controlled loads at the
\end{abstract}

CrossCheck date: 23 October 2017

Received: 26 November 2016/Accepted: 24 October 2017/Published online: 9 January 2018

(C) The Author(s) 2018. This article is an open access publication

$\bowtie$ Yunfei MU

yunfeimu@tju.edu.cn

Mingshen WANG

wangmingshen@tju.edu.cn

Tao JIANG

tjiang@nedu.edu.cn

Hongjie JIA

hjjia@tju.edu.cn

Xue LI

xli@nedu.edu.cn

Kai HOU

hdbhyj@tju.edu.cn

Tong WANG

wangtong@sgepri.sgcc.com.cn

1 Key Laboratory of Smart Grid of Ministry of Education, Tianjin University, Tianjin 300072, China

2 Department of Electrical Engineering, Northeast Electric Power University, Jilin 132012, China

3 NARI Solar Energy Technologies Company Limited, Nanjing 210009, China demand side, are able to serve as the energy storage system to smooth the load fluctuations. However, it is a challenge to properly model different types of DSRs. To solve this problem, a unified state model is first developed to describe the characteristics of different DSRs. Then a load curve smoothing strategy is proposed to offset the load fluctuations of the tie-line of the residential region, where a control matrix deduced from the unified state model is introduced to manage the power outputs of different DSRs, considering the response order and the comfort levels. Finally, a residential region with households is used to validate the load curve smoothing strategy based on the unified state model, and the results show that the power fluctuation rate of the tie-line is significantly decreased. Meanwhile, comparative study results are shown to demonstrate the advantages of the unified state model based load curve smoothing strategy.

Keywords Demand side resource (DSR), Power fluctuation, Distributed generation (DG), Electric vehicle (EV), Thermostatically-controlled load (TCL), Load curve smoothing

\section{Introduction}

The renewable energy resources, such as wind and solar, have aroused more and more attention around the world due to their renewable and environmental advantages in recent years [1]. However, renewable distributed generation (DG) with intermittent characteristics will introduce a large amount of uncertainties to the demand side. One concern is the severe variations to the load power of the tieline in an electric region [2], which will significantly impact the stable operation of the power grid and limit the utilization of the renewable energy [3]. 
One of the traditional control strategies for the load curve smoothing of the tie-line is the regulation of the traditional generators [4]. However, the response speed of these types of generators is not fast enough to follow the power fluctuations of DGs, and the efficiency of power generation will be reduced. Another method to tackle this problem is through the utilization of energy storage system (ESS), such as batteries, flywheels, etc., to respond to the power fluctuations [5, 6]. The ESS was used to improve the voltage profile which was affected by the stochastic outputs of the DGs [7, 8]. The ESS was investigated for peak load shaving of the tie-line in $[9,10]$. Through dynamic adjusting of the charging power, the ESS is able to effectively respond to the power fluctuations caused by the DGs $[11,12]$. However, large scale adoption of ESS has not economical and practical until now.

With the fast development of a smart grid, there have been growing interests in the flexibility of demand response. The total response capacity from different types of demand side resources (DSRs) is considerable [13, 14]. Among all the DSRs, the DG is able to decrease its power generation [15], and the electric vehicle (EV) is able to operate as a mobile energy storage unit with its rapid charging and discharging capabilities [16], and the thermostatically-controlled load (TCL) can respond to the power variations by switching between "ON" and "OFF" [17]. These available DSRs with different response characteristics have been proven to be effective resources for demand response at the demand side. Thus, a number of DSRs are able to provide various types of ancillary services for the power grid. Considering the response capacity from the EVs [16] and TCLs [17], these resources were able to mitigate the voltage variations in the distribution network with DGs. Meanwhile, the power loss of the distribution network was minimized based on a real-time coordination of the EV charging load [18, 19]. The EVs were able to operate as an EES to reshape the load profile and alleviate the peak load relying on their rapid responding capability $[20,21]$. The TCLs, such as heat pumps, were able to smooth the power fluctuations of the tie-line with DGs [22, 23].

The above researches focused on improving the power quality using a single type of DSR. However, each type of DSR has its own response characteristics and the mathematical model of each DSR is quite different. As a result, it is difficult for an aggregator to coordinate different types of DSRs for the operational management at the demand side.

At present, in order to realize the coordinated control of different types of DSRs, an aggregator is usually built for each type of DSR. In [24], the EV aggregator and the TCL aggregator were established separately to provide frequency response service to the power grid, and the control signal was first responded by the EV aggregator and then the remaining unbalanced power was further compensated by the TCL aggregator. However, the following two aspects need to be further investigated:

1) In the existing researches, one aggregator only focuses on one type of DSR.

2) For different aggregators with different types of DSRs, the queuing control strategy is usually used to realize the power control of the different aggregators.

As a result, these two aspects in fact increase the difficult to realize the coordinated control of different types of DSRs, which also means that the response characteristics of different types of DSRs are not fully utilized. The existing control strategies usually lead to the excessive dependence on one type of DSR and the control effect is poor and the response capacity is limited during some of the time periods. Thus it is necessary to develop a unified mathematical model to describe their energy storage capability and their response characteristics. And an aggregator is able to manage different types of DSRs based on the unified state model, which contributes to realizing the coordinated control of different types of DSRs.

In this paper, a load curve smoothing strategy based on a unified state model of different DSRs is developed for the demand response. Based on the state models of different DSRs, a unified state model is first developed to describe the response characteristics of the DSRs with a unified mathematical expression. Then, a load curve smoothing strategy using the unified state model is proposed to coordinate different types of DSRs to smooth the power fluctuations caused by the DGs. A control matrix is introduced to manage the power outputs of different DSRs, where the comfort levels of the power consumers and the response order are considered. The power fluctuation rate is introduced as an indicator to evaluate the load power variations of the tie-line in a residential region. The unified state model in the proposed load curve smoothing strategy can determine the real-time response capacity and the response states of different types of DSRs, and the complexity for managing large quantities of DSRs is significantly reduced. Compared with the existing researches, comparative studies show that the unified state model based load curve smoothing strategy takes better advantage of the response characteristics of different types of DSRs and has a better smoothing control effect.

\section{Framework of load curve smoothing strategy}

Considering the response capability from different types of DSRs, the framework of the load curve smoothing strategy for a given residential region is shown in Fig. 1. In 
this paper, the power generation of DGs, the charging/ discharging power of EVs and the consumed power of the TCLs are uniformly defined as the power outputs of the DSRs. The power output of the power generation and discharging power is a positive value, and the power output of the charging power and consumed power is a negative value.

In the load curve smoothing strategy, the load data of the tie-line in the residential region, which is the sum of the uncontrollable load, the DGs, the EVs and the TCLs, is measured in real time. To implement the smoothing control strategy, the real-time power demand of the region is determined according to the power fluctuations of the load. The power demand is compensated by the DSRs.

An aggregator is responsible for managing the power outputs of all the available DSRs. The DSRs of the DGs, EVs and TCLs are able to serve as an ESS under the coordinated control of the aggregator. The aggregator is able to obtain the operational information of the available DSRs, evaluate their response capacity and determine their power outputs with the unified state model. And the required operational information of different types of DSRs is fully discussed in Sect. 3. Compared with the response capacity of a single DSR, the aggregated capacity of all DSRs managed by the aggregator is significantly increased.

According to the power demand in the residential region and the actual response capacity from the DSRs, the power outputs of all the DSRs are determined and the control signals are allocated to all the available DSRs. The DSRs accept the control signals from the aggregator, and the power outputs of these resources are adjusted accordingly to respond to the power demand.

\section{Unified state model of DSRs}

Each type of DSR at the demand side has its own response characteristic. The unified state model is able to describe their response characteristics with a unified mathematical expression. The DSRs of DGs, EVs and TCLs are investigated in this section.

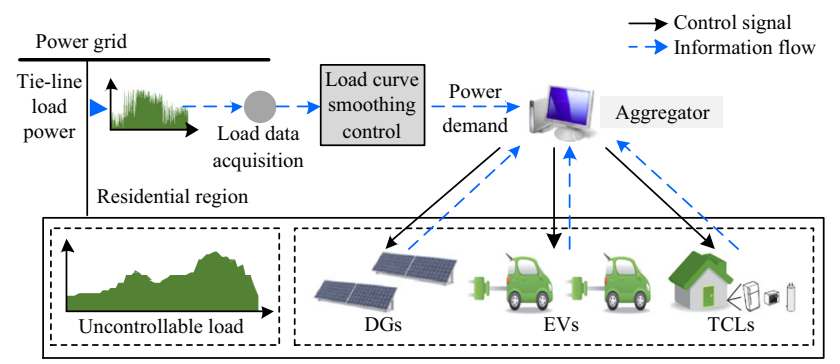

Fig. 1 Framework of the load curve smoothing strategy

\subsection{State model of DG}

The DGs, such as the rooftop photovoltaic panels and the wind turbines, inject power to the power grid. The operational area of an individual DG is shown in Fig. 2.

The $\underline{P}_{g, t}^{G}$ and $\bar{P}_{g, t}^{G}$ of DG $g$ are expressed by (1).

$\left\{\begin{array}{l}P_{g, t}^{G}=0 \\ \bar{P}_{g, t}^{G}=P_{g, t}^{G m}\end{array}\right.$

where $P_{g, t}^{G m}$ is the maximum power generation of DG $g$ at time point $t ; \underline{P}_{g, t}^{G}$ and $\bar{P}_{g, t}^{G}$ are lower and upper limitations of power output of DG $g$ at time point $t$.

The state model of the DG is given by (2).

$E_{g, t+\Delta t}^{G}=E_{g, t}^{G}+\frac{P_{g, t}^{G} \Delta t}{Q_{g}^{G}}=E_{g, t}^{G}+P_{g, t}^{G} \frac{\Delta t}{Q_{g}^{G}}$

where $E_{g, t}^{G}$ is the state of DG $g$ at time point $t$ varies within $[0,1] ; E_{g, t+\Delta t}^{G}$ is the predicted state value at time point $t+\Delta t ; P_{g, t}^{G}$ is the power generation of DG $g$ at time point $t$; $Q_{g}^{G}$ is the accumulated energy generated by the DG with the rated power generation, which is calculated by (3).

$Q_{g}^{G}=P_{g}^{G r} \Delta t M$

where $P_{g}^{G r}$ is the rated power generation of DG $g ; M$ is the number of time intervals in a day.

To build the state model of the DG, the operational information of $E_{g, t}^{G}, P_{g, t}^{G}, P_{g, t}^{G m}$ and $P_{g}^{G r}$ needs to be obtained by the aggregator.

\subsection{State model of EV}

When traveling, an EV makes no contribution to the power grid. When connected to the power grid, a bi-directional power flow (charging/discharging) between the $\mathrm{EV}$ and the power grid can be realized.

The $\underline{P}_{v, t}^{V}$ and $\bar{P}_{v, t}^{V}$ of EV $v$ are expressed by (4).

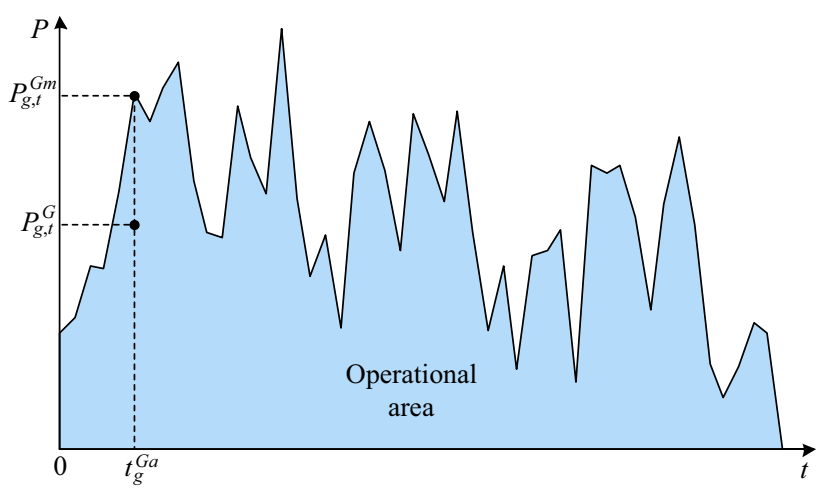

Fig. 2 Operational area of an individual DG 
$\begin{cases}\underline{P}_{v, t}^{V}=-P_{v}^{V c}, \bar{P}_{v, t}^{V}=P_{v}^{V d} & t \in\left[t_{v}^{V s}, t_{v}^{V d}\right] \\ \underline{P}_{v, t}^{V}=\bar{P}_{v, t}^{V}=0 & t \notin\left[t_{v}^{V s}, t_{v}^{V d}\right]\end{cases}$

where $\underline{P}_{v, t}^{V}$ and $\bar{P}_{v, t}^{V}$ are lower and upper limitations of power output of EV $v$ at time point $t, \bar{P}_{v, t}^{V}$ is assumed to be equal to $-\underline{P}_{v, t}^{V} ; P_{v}^{V c}$ and $P_{v}^{V d}$ are rated charging and discharging power of EV $v ; t_{v}^{V s}$ and $t_{v}^{V d}$ are start and finish charging times of EV $v$.

The operational area of an individual EV is illustrated in the shaded area shown in Fig. 3, which is limited by the power output limitations and the state of charge (SOC) limitations. Points $A, B, C, D, E$ and $F$ are used to obtain the upper and lower boundaries of the operational area. The upper boundary of the operational area follows the path of " $A-B-C$ ", on which the EV is charged with the rated charging power from $t_{v}^{V s}$ until the $\overline{S O C}_{v}^{V}$ is reached, where $\overline{S O C}_{v}^{V}$ is upper limitation of SOC value of EV $v$. The lower boundary of the operational area follows the path of " $A-D$ $E-F$ ", on which the $\mathrm{EV}$ is discharged at the rated discharging power from $t_{v}^{V s}$ until the $\underline{S O C}_{v}^{V}$ is reached (from point $A$ to $D$ ) and the $\mathrm{EV}$ is charged at the rated charging power (from point $E$ to $F$ ) to achieve the $S O C_{v}^{V d}$ at $t_{v}^{V d}$, where $\underline{S O C}_{v}^{V}$ is lower limitation of SOC value of EV $v$ and $S O C_{v}^{V d}$ is the expected SOC value for traveling of EV $v$.

The $S_{v, t}^{V}$ is given by (5). When connected to the power grid, the state model of the EV is given by (6).

$S_{v, t}^{V}=\frac{S O C_{v, t}^{V}-\underline{S O C}_{v}^{V}}{S_{\mathcal{S O C}}^{V}-\underline{S O C}_{v}^{V}}$

$S_{v, t+\Delta t}^{V}=S_{v, t}^{V}-\frac{P_{v, t}^{V} \Delta t}{Q_{v, t}^{V r}}=S_{v, t}^{V}+P_{v, t}^{V}\left(-\frac{\Delta t}{Q_{v, t}^{V r}}\right)$

where $S_{v, t}^{V}$ is the normalized SOC value of EV $v$ at time point $t$ varies within $[0,1]$; $S O C_{v, t}^{V}$ is the SOC value of $\mathrm{EV} v$ at time point $t ; S_{v, t+\Delta t}^{V}$ is the predicted value at time point

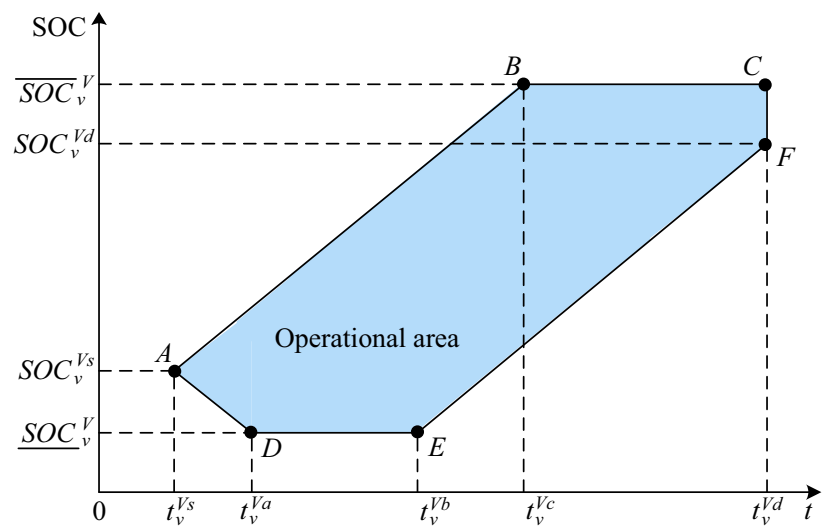

Fig. 3 Operational area of an individual EV
$t+\Delta t ; P_{v, t}^{V}$ is the power output of EV $v$ at time point $t ; Q_{v, t}^{V r}$ is determined by (7).

$Q_{v, t}^{V r}=\left\{\begin{array}{cl}\frac{Q_{v}^{V}}{\eta_{v}^{V c}\left(\overline{S O C}_{v}^{V}-\underline{S O C}_{v}^{V}\right)} & P_{v, t}^{V}<0 \\ Q_{v}^{V}\left(\overline{S O C}_{v}^{V}-\underline{S O C}_{v}^{V}\right) & P_{v, t}^{V}=0 \\ Q_{v}^{V} \eta_{v}^{V d}\left(\overline{S O C}_{v}^{V}-\underline{S O C}_{v}^{V}\right) & P_{v, t}^{V}>0\end{array}\right.$

where $Q_{v}^{V}$ is the battery capacity of $\mathrm{EV} v ; \eta_{v}^{V c}$ and $\eta_{v}^{V d}$ are charging and discharging efficiencies of EV $v$.

To build the state model of the EV, the operational information of $S_{v, t}^{V}, P_{v, t}^{V}, P_{v, t}^{V c}, P_{v, t}^{V d}, Q_{v}^{V}, \eta_{v}^{V c}, \eta_{v}^{V d}, t_{v}^{V d}, S O C_{v}^{V d}$, $\underline{S O C}_{v}^{V}$ and $\overline{S O C}_{v}^{V}$ needs to be obtained by the aggregator.

\subsection{State model of TCL}

The power consumption of TCLs should be modeled to satisfy the expected temperature requirements of the consumers. Taking the TCL of the heat pump for heating as an example, the operational area of an individual TCL is shown in Fig. 4.

The $\underline{P}_{l, t}^{L}$ and $\bar{P}_{l, t}^{L}$ of TCL $l$ are expressed by (8).

$\left\{\begin{array}{l}\underline{P}_{l, t}^{L}=-P_{l, t}^{L c} \\ \bar{P}_{l, t}^{L}=0\end{array}\right.$

where $\underline{P}_{l, t}^{L}$ and $\bar{P}_{l, t}^{L}$ are lower and upper limitations of power output of TCL $l$ at time point $t ; P_{l, t}^{L c}$ is the rated power consumption of TCL $l$ at time point $t$.

As depicted in Fig. 4, during the time period of $\left[0, t_{l}^{L b}\right]$, the TCL operates with the "ON" state and the room temperature is increasing. During the time period of $\left[t_{l}^{L b}, t_{l}^{L d}\right]$, the TCL operates in the "OFF" state and the room temperature is decreasing. The lower and upper thermostat settings of TCL $\underline{T}_{l}^{L c o n}$ and $\bar{T}_{l}^{L c o n}$ are defined to obtain the controllable area. For the TCLs in the "ON" group, the TCLs within the range of $\left[\underline{T}_{l}^{L c o n}, \bar{T}_{l}^{L}\right]$ can be selected to turn "OFF", where $\bar{T}_{l}^{L}$ is the upper limitations of room

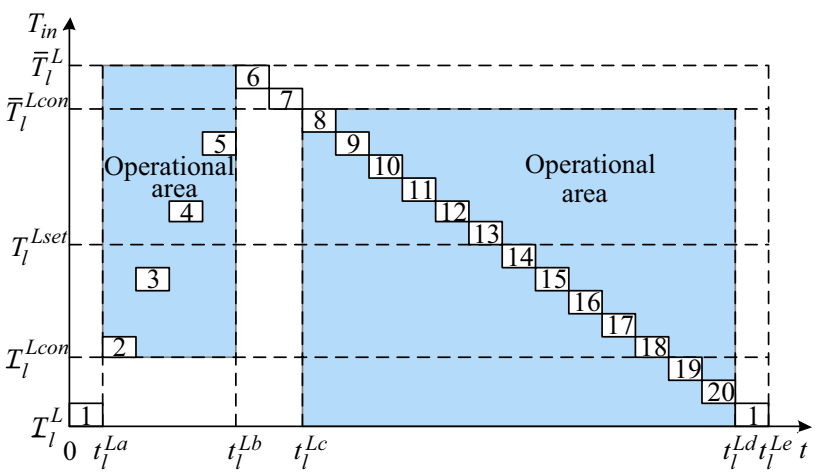

Fig. 4 Operational area of an individual TCL 
temperature of TCL $l$. Similarly, for the TCLs in the "OFF" state, the TCLs within the the range of $\left[\underline{T}_{l}^{L}, \bar{T}_{l}^{L c o n}\right]$ can be selected to turn "ON", where $\underline{T}_{l}^{L}$ is the lower limitations of room temperature of TCL $l$. The operational area will help to decrease the switching frequency of the TCL.

In order to standardize the $T_{l, t}^{L}$ and $T_{l, t}^{L o u t}, \theta_{l, t}^{L}\left(0 \leq \theta_{l, t}^{L} \leq 1\right)$ and $\theta_{l, t}^{\text {Lout }}$ are the normalized room temperature and the outdoor temperature of TCL respectively, which are determined by (9). The state model of the TCL is then given by (10).

$$
\begin{aligned}
& \left\{\begin{array}{l}
\theta_{l, t}^{L}=\frac{T_{l, t}^{L}-\underline{T}_{l}^{L}}{\bar{T}_{l}^{L}-\underline{T}_{l}^{L}} \\
\theta_{l, t}^{\text {Lout }}=\frac{T_{l, t}^{L o u t}-\underline{T}_{l}^{L}}{\bar{T}_{l}^{L}-\underline{T}_{l}^{L}}
\end{array}\right. \\
& \theta_{l, t+\Delta t}^{L}=\left[\theta_{l, t}^{\text {Lout }}+\left(\theta_{l, t}^{L}-\theta_{l, t}^{L o u t}\right) a_{l}\right]-P_{l, t}^{L} \frac{1-a_{l}}{\bar{T}_{l}^{L}-\underline{T}_{l}^{L}}= \\
& {\left[\theta_{l, t}^{\text {Lout }}+\left(\theta_{l, t}^{L}-\theta_{l, t}^{\text {Lout }}\right) a_{l}\right]+P_{l, t}^{L} \frac{a_{l}-1}{\bar{T}_{l}^{L}-\underline{T}_{l}^{L}}}
\end{aligned}
$$

where $\theta_{l, t+\Delta t}^{L}$ is the predicted state value at time point $t+\Delta t ; a_{l}$ is equal to $\mathrm{e}^{\Delta t /\left(R_{l} C_{l}\right)} ; R_{l}$ and $C_{l}$ are the thermal resistance and capacitance of TCL $l ; P_{l, t}^{L}$ is equal to $\underline{P}_{l, t}^{L}$ in the "ON" state and $\bar{P}_{l, t}^{L}$ in the "OFF" state; $T_{l, t}^{L}$ and $T_{l, t}^{L \text { out }}$ are the room temperature and the outdoor temperature of TCL $l$ at time point $t$.

To acquire the state model of the TCL, the operational information of $P_{l, t}^{L}, P_{l, t}^{L c}, R_{l}, C_{l}, \underline{T}_{l}^{L}, \bar{T}_{l}^{L}, \underline{T}_{l}^{L c o n}, \bar{T}_{l}^{L c o n}$ and $T_{l, t}^{\text {Lout }}$ needs to be obtained by the aggregator.

\subsection{Unified state model}

According to the state models in Sects. 3.1, 3.2 and 3.3, the (2), (6) and (10) have a unified mathematical expression. Then the state model of different DSRs is given by (11), which is further explained by (12)-(17).

$$
\begin{aligned}
& {\left[\begin{array}{c}
\boldsymbol{E}_{t+\Delta t}^{G} \\
\boldsymbol{S}_{t+\Delta t}^{V} \\
\boldsymbol{\theta}_{t+\Delta t}^{L}
\end{array}\right]=\left[\begin{array}{c}
\boldsymbol{E}_{t}^{G} \\
\boldsymbol{S}_{t}^{V} \\
\boldsymbol{\theta}_{t}^{L}
\end{array}\right]+\left[\begin{array}{ccc}
\boldsymbol{P}_{t}^{G} & \mathbf{0} & \mathbf{0} \\
\mathbf{0} & \boldsymbol{P}_{t}^{V} & \mathbf{0} \\
\mathbf{0} & \mathbf{0} & \boldsymbol{P}_{t}^{L}
\end{array}\right]\left[\begin{array}{c}
\boldsymbol{\delta}_{t}^{G} \\
\boldsymbol{\delta}_{t}^{V} \\
\boldsymbol{\delta}_{t}^{L}
\end{array}\right]} \\
& \left\{\begin{array}{l}
\boldsymbol{E}_{t+\Delta t}^{G}=\left[E_{1, t+\Delta t}^{G}, \ldots, E_{g, t+\Delta t}^{G}, \ldots, E_{N_{G}, t+\Delta t}^{G}\right]^{\mathrm{T}} \\
\boldsymbol{S}_{t+\Delta t}^{V}=\left[S_{1, t+\Delta t}^{V}, \ldots, S_{v, t+\Delta t}^{V}, \ldots, S_{N_{V}, t+\Delta t}^{V}\right]^{\mathrm{T}} \\
\boldsymbol{\theta}_{t+\Delta t}^{L}=\left[\theta_{1, t+\Delta t}^{L}, \ldots, \theta_{l, t+\Delta t}^{L}, \ldots, \theta_{N_{L}, t+\Delta t}^{L}\right]^{\mathrm{T}}
\end{array}\right. \\
& \boldsymbol{E}_{t}^{G}=\left[E_{1, t}^{G}, \ldots, E_{g, t}^{G}, \ldots, E_{N_{G}, t}^{G}\right]^{\mathrm{T}}
\end{aligned}
$$

$$
\begin{aligned}
& \boldsymbol{S}_{t}^{V}=\left[S_{1, t}^{V}, \ldots, S_{v, t}^{V}, \ldots, S_{N_{V}, t}^{V}\right]^{\mathrm{T}} \\
& \boldsymbol{\theta}_{t}^{L}=\left[\begin{array}{c}
\theta_{1, t}^{\text {out }}+\left(\theta_{1, t}^{L}-\theta_{1, t}^{\text {out }}\right) a_{1} \\
\vdots \\
\theta_{l, t}^{\text {out }}+\left(\theta_{l, t}^{L}-\theta_{l, t}^{\text {out }}\right) a_{l} \\
\vdots \\
\theta_{N_{L}, t}^{\text {out }}+\left(\theta_{N_{L}, t}^{L}-\theta_{N_{L}, t}^{\text {out }}\right) a_{N_{L}}
\end{array}\right] \\
& \left\{\begin{aligned}
\boldsymbol{P}_{t}^{G} & =\operatorname{diag}\left[P_{1, t}^{G}, \ldots, P_{g, t}^{G}, \ldots, P_{N_{G}, t}^{G}\right] \\
\boldsymbol{P}_{t}^{V} & =\operatorname{diag}\left[P_{1, t}^{V}, \ldots, P_{v, t}^{V}, \ldots, P_{N_{V}, t}^{V}\right] \\
\boldsymbol{P}_{t}^{L} & =\operatorname{diag}\left[P_{1, t}^{L}, \ldots, P_{l, t}^{L}, \ldots, P_{N_{L}, t}^{L}\right]
\end{aligned}\right. \\
& \left\{\begin{array}{l}
\boldsymbol{\delta}_{t}^{G}=\left[\frac{\Delta t}{Q_{1}^{G}}, \ldots, \frac{\Delta t}{Q_{g}^{G}}, \ldots, \frac{\Delta t}{Q_{N_{G}}^{G}}\right]^{\mathrm{T}} \\
\boldsymbol{\delta}_{t}^{V}=\left[-\frac{\Delta t}{Q_{1}^{V r}}, \ldots,-\frac{\Delta t}{Q_{v}^{V r}}, \ldots,-\frac{\Delta t}{Q_{N_{V}}^{V r}}\right]^{\mathrm{T}}
\end{array}\right. \\
& \boldsymbol{\delta}_{t}^{L}=\left[\frac{a_{1}-1}{\bar{T}_{1}^{L}-\underline{T}_{1}^{L}}, \ldots, \frac{a_{l}-1}{\bar{T}_{l}^{L}-\underline{T}_{l}^{L}}, \ldots, \frac{a_{N_{L}}-1}{\bar{T}_{N_{L}}^{L}-\underline{T}_{N_{L}}^{L}}\right]^{\mathrm{T}}
\end{aligned}
$$

where $N_{G}$ is the number of DGs; $N_{V}$ is the number of EVs; $N_{L}$ is the number of TCLs.

Based on the state model given by (11), the unified state model is given by (18).

$\boldsymbol{x}(t+\Delta t)=\boldsymbol{x}(t)+\boldsymbol{P}(t) \boldsymbol{\delta}(t)$

where $\boldsymbol{x}$ is the state matrix of DSRs; $\boldsymbol{P}$ is the power output matrix of DSRs; $\boldsymbol{\delta}$ is the time interval matrix after correction of DSRs.

\section{Load curve smoothing strategy}

\subsection{Steps of load curve smoothing strategy}

Based on (18), a load curve smoothing strategy is developed to smooth the load power fluctuations of the tieline.

The $r^{T}$, determined by (19) and (20), is used as an indicator to evaluate the power fluctuations.

$r^{T}=f^{T}\left(\frac{P_{\max }^{D}-P_{\min }^{D}}{P_{\text {rated }}^{D}}\right)$
$\left\{\begin{array}{l}P_{\max }^{D}=\max \left(P_{t}^{D}, P_{t-\Delta t}^{D}, \ldots, P_{t-(n-1) \Delta t}^{D}\right) \\ P_{\min }^{D}=\min \left(P_{t}^{D}, P_{t-\Delta t}^{D}, \ldots, P_{t-(n-1) \Delta t}^{D}\right) \\ T=n \Delta t\end{array}\right.$ 
where $f^{T}(\cdot)$ is a function to calculate the power fluctuation rate of the load during the time period $T ; P_{\text {rated }}^{D}$ is the rated load value; $P_{\min }^{D}$ and $P_{\max }^{D}$ are the minimum and maximum load values of the tie-line during the time period $T$, respectively; $P_{t}^{D}$ is the load power of tie-line at time point $t$.

The $r_{t}$, determined by (21), is used to evaluate the realtime power fluctuation.

$r_{t}=\frac{P_{t}^{D}-P_{t-\Delta t}^{D}}{\Delta t}$

The $\delta_{\text {drop }}$ and $\delta_{\text {rise }}$, which are used to limit the variations of $r_{t}$, are further defined by (22).

$\left\{\begin{array}{l}\delta_{\text {drop }}=-\frac{P_{\text {rated }}^{D} r_{\text {limit }}^{T}}{T} \\ \delta_{\text {rise }}=\frac{P_{\text {rated }}^{D} r_{\text {limit }}^{T}}{T}\end{array}\right.$

where $r_{\text {limit }}^{T}$ is the limitation of power fluctuation rate during time period $T$.

Based on the unified state model and the definition of the power fluctuation rate, the following steps are used to implement the load curve smoothing control strategy.

Step 1 Determining target power change of the load.

The following rules are used to determine the target smoothing load power of tie-line $P_{t}^{*}$.

1) If $r_{t}<\delta_{\text {drop }}$, where $\delta_{\text {drop }}$ is the drop rate limitations of power fluctuation rate, then

$P_{t}^{*}=P_{t-\Delta t}^{D}+\delta_{\text {drop }} \Delta t$

2) If $r_{t}>\delta_{\text {rise }}, \delta_{\text {rise }}$ is the rise rate limitations of power fluctuation rate, then

$P_{t}^{*}=P_{t-\Delta t}^{D}+\delta_{\text {rise }} \Delta t$

3) If $\delta_{\text {drop }} \leq r_{t} \leq \delta_{\text {rise }}$, then

$P_{t}^{*}=P_{t}^{D}$

Thus, $\Delta P_{t}^{*}$ is determined by (26).

$\Delta P_{t}^{*}=P_{t}^{*}-P_{t}^{D}$

where $\Delta P_{t}^{*}$ is the target power change of tie-line, which needs to be balanced by the DSRs considering their response capacity. If $\Delta P_{t}^{*}$ is higher (lower) than zero, then the chosen DSRs have to increase (decrease) their power outputs to accomplish the target smoothing load value.

Step 2 Determining response capacity of DSRs.

To realize the load curve smoothing control with the unified state model, the $\boldsymbol{P}(t)$ given by (16) is decomposed into $\boldsymbol{B P}(t)$, i.e., the real-time power output $P_{j j}^{i}(t)$ is replaced by $B_{j j}^{i} \bar{P}_{j j}^{i}(t)$, as given by (27).

$\left\{\begin{array}{l}\boldsymbol{P}(t)=\boldsymbol{B} \boldsymbol{P}(t) \\ P_{j j}^{i}(t)=B_{j j}^{i} \bar{P}_{j j}^{i}(t)\end{array}\right.$

where $\boldsymbol{B}$ is the power output control matrix of DSRs; $\overline{\boldsymbol{P}}(t)$ is the upper power output matrix of DSRs; $i$ denotes the types of DSRs, $i \in\{G, V, L\}$; element $\bar{P}_{j j}^{i}(t)$ of $\overline{\boldsymbol{P}}(t)$ is the upper power output limitation of DSR $j$; element $B_{j j}^{i}$ of $\boldsymbol{B}$ $\left(B_{j j}^{G} \in[0,1], B_{j j}^{V} \in[-1,1], B_{j j}^{L} \in\{-1,1\}\right)$ is defined as the power output control variable, which is used to increase or decrease the power output of DSR $j$.

Then the revised unified state model is given by (28), which can be used to manage and control the power output of all the DSRs.

$\boldsymbol{x}(t+\Delta t)=\boldsymbol{x}(t)+\boldsymbol{B P}(t) \boldsymbol{\delta}(t)$

The response order of the DSR is determined by the row order in the unified state model, which is updated with time. For example, the response order of the DSRs at row $m$ and $n$ should be exchanged: (1) for the column matrix $(\boldsymbol{x}(t)$ and $\boldsymbol{\delta}(t))$ in (28), the element at row $m$ is exchanged with the element at row $n$; (2) for the diagonal matrix (B and $\overline{\boldsymbol{P}}(t))$ in (28), the diagonal element at row $m$ is exchanged with the diagonal element at row $n$. The response order of the DSRs is determined according to the different dispatching costs of different DSRs or other response orders preset by the aggregator. The response order of the DSRs for the smoothing control utilized in this paper is discussed in Sect. 4.2.

Based on the unified state model, the response capacity for increasing power output $\boldsymbol{P}^{\mathrm{up}}(t)$ is given by (29), and the response capacity for decreasing power output $\boldsymbol{P}^{\mathrm{dn}}(t)$ is given by (30). The $\boldsymbol{P}^{\mathrm{up}}(t)$ is an $N \times 1$ matrix, and the nonnegative element at row $m$ indicates the response capacity of the $m^{\text {th }}$ resource for increasing power output. The $\boldsymbol{P}^{\mathrm{dn}}(t)$ is also an $N \times 1$ matrix, and the non-positive element at row $m$ indicates the response capacity of the $m^{\text {th }}$ resource for decreasing power output.

$\boldsymbol{P}^{\text {up }}(t)=(\overline{\boldsymbol{B}}-\boldsymbol{B}) \overline{\boldsymbol{P}}(t) \boldsymbol{L}_{0}$

where the diagonal elements of $\overline{\boldsymbol{B}}$ are the maximum values of the control variables; $\boldsymbol{L}_{0}$ is an $N \times 1$ matrix with each element equal to 1 .

$\boldsymbol{P}^{\mathrm{dn}}(t)=(\underline{\boldsymbol{B}}-\boldsymbol{B}) \overline{\boldsymbol{P}}(t) \boldsymbol{L}_{0}$

where the diagonal elements of $\underline{\boldsymbol{B}}$ are the minimum values of the control variables.

To introduce the response capability of the state model, the lower triangular matrix $\overline{\boldsymbol{B}}^{*}$ and $\underline{\boldsymbol{B}}^{*}$ are defined to evaluate the response capacity for increasing and 
decreasing power output. The elements of $\overline{\boldsymbol{B}}^{*}$ and $\underline{\boldsymbol{B}}^{*}$ are defined by (31) and (32).

$\bar{B}_{j k}^{*}=\left\{\begin{array}{cc}0 & j<k \\ \bar{B}_{j j} & j \geq k\end{array}\right.$

$\underline{B}_{i j}^{*}=\left\{\begin{array}{cc}0 & j<k \\ \underline{B}_{j j} & j \geq k\end{array}\right.$

Then the response capacity for increasing and decreasing power output is further given by (33).

$$
\left\{\begin{array}{l}
\boldsymbol{P}^{\mathrm{up} *}(t)=\left(\overline{\boldsymbol{B}}^{*}-\boldsymbol{B}\right) \overline{\boldsymbol{P}}(t) \\
\boldsymbol{P}^{\mathrm{dn} *}(t)=\left(\underline{\boldsymbol{B}}^{*}-\boldsymbol{B}\right) \overline{\boldsymbol{P}}(t)
\end{array}\right.
$$

where $\boldsymbol{P}^{\text {up* }}(t)$ is an $N \times 1$ matrix, and the non-negative element at row $m$ indicates the response capacity of the $1 \sim m$ resources for increasing power output; $\boldsymbol{P}^{\mathrm{dn} *}(t)$ is an $N \times 1$ matrix, and the non-positive element at row $m$ indicates the response capacity of the $1 \sim m$ resources for decreasing power output.

Step 3 Determining the actual control matrix $\boldsymbol{B}^{*}$.

1) If $\Delta P_{t}^{*}>0$, obtain the maximum subscript $j_{1}$ of $P_{j_{1}, 1}^{\text {up } *}$ which satisfies $P_{j_{1}, 1}^{\mathrm{up} *} \leq \Delta P_{t}^{*}$. And the $1 \sim j_{1}$ resources in the unified state model have to increase their power output to realize the active power change $\Delta P_{t}^{*}$ as given by (34).

$$
\left\{\begin{array}{l}
B_{j k}^{*}=\bar{B}_{j k} \quad j=k, j \leq j_{1} \\
B_{j k}^{*}=B_{j k} \quad j=k, j>j_{1} \\
B_{j k}^{*}=0 \quad j \neq k
\end{array}\right.
$$

2) If $\Delta P_{t}^{*}=0$, there is no need to adjust the element values of $\boldsymbol{B}$ as given by (35).

$\boldsymbol{B}^{*}=\boldsymbol{B}$

3) If $\Delta P_{t}^{*}<0$, obtain the maximum subscript $j_{2}$ of $P_{j_{2}, 1}^{\mathrm{dn} *}$ which satisfies $P_{j_{2}, 1}^{\mathrm{up} *} \geq \Delta P_{t}^{*}$. The $1 \sim j_{2}$ resources in the unified state model have to decrease their power output to realize the negative power change $\Delta P_{t}^{*}$ as given by (36).

$$
\left\{\begin{array}{cl}
B_{j k}^{*}=\underline{B}_{j k} & j=k, j \leq j_{2} \\
B_{j k}^{*}=B_{j k} & j=k, j>j_{2} \\
B_{j k}^{*}=0 & j \neq k
\end{array}\right.
$$

Thus the power outputs of all the DSRs are determined by (37). Meanwhile, the state model of the DSRs is updated by (38), and the row order of the unified state model is updated with the response order of the DSRs.

$$
\begin{aligned}
& \boldsymbol{P}(t)=\boldsymbol{B}^{*} \overline{\boldsymbol{P}}(t) \\
& \boldsymbol{x}(t+\Delta t)=\boldsymbol{x}(t)+\boldsymbol{P}(t) \boldsymbol{\delta}(t)=\boldsymbol{x}(t)+\boldsymbol{B}^{*} \overline{\boldsymbol{P}}(t) \boldsymbol{\delta}(t)
\end{aligned}
$$

The advantages of the unified state model for load curve smoothing control primarily include three parts. First, different types of DSRs are able to be described in the unified state model, which provide a new solution for managing the large number of DSRs in the aggregator with different types of DSRs. Secondly, once the required response capacity is determined, the new power output control matrix is correspondingly generated to manage the power outputs of the DSRs in real time. Thirdly, the states of the DSRs at the next time step are able to be predicted and obtained, which will be used for short-time prediction of DSRs.

\subsection{Response order for smoothing control}

In this paper, it is assumed that the connecting states of the EVs include the charging state $\left(P_{j, t}^{V}=-P_{j, t}^{V c}\right)$, the idle state $\left(P_{j, t}^{V}=0\right)$ and the discharging state $\left(P_{j, t}^{V}=P_{j, t}^{V d}\right)$. It is also assumed that the connecting states of the TCLs include the "ON" state $\left(P_{j, t}^{L}=-P_{j, t}^{L c}\right)$ and the "OFF" state $\left(P_{j, t}^{L}=0\right)$. To respond to the power demand, the controllable EV can change its connecting state between charging state and idle state ("charging $\leftrightarrow$ idle") and between idle state and discharging state ("idle $\leftrightarrow$ discharging"), and the controllable TCL can change its connecting state between "ON" state and "OFF" state ("ON $\leftrightarrow$ OFF"). The response modes of "charging $\leftrightarrow$ idle" and "ON $\leftrightarrow$ OFF" are just to change the time period for power consumption, and the cost of the EV's "charging $\leftrightarrow$ idle" mode is verified to be lower than the cost of TCL's "ON $\leftrightarrow$ OFF" mode [25]. However, the EV battery has to discharge power to the power grid in the response mode of "idle $\leftrightarrow$ discharging", which requires more cost compensation than other response modes [26].

In order to utilize the unified state model in the smoothing control, the response order of the DSRs for increasing and decreasing power output is shown in Fig. 5. The structure of both Fig. $5 \mathrm{a}$ and $5 \mathrm{~b}$ is divided into three layers. The first layer introduces the response order of the DSR type during the load curve smoothing. In the second layer, each column represents the response order of the DSRs with the corresponding DSR type. In the third layer, each unit provides the satisfied constraints of the corresponding DSRs. And the response order of Fig. 5a and 5b is then introduced in detail. Two main principles of the cost of demand response and the utilization level of renewable energy are considered when deciding the response order of the DSRs.

As shown in Fig. 5a, the response order of the DSRs for increasing power output is the charging EVs to stop charging, the "ON" state TCLs to switch off, the idle EVs to discharge and then the uncontrollable DSRs. The response order for increasing power output is shown as 
follows: (1) the charging EV with higher state value will have the priority to stop charging; (2) the "ON" state TCL with higher state value will have the priority to switch off; (3) the idle EV with higher state value will have the priority to discharge; (4) the DSRs, which have reached their upper power output limitations can be seen as the uncontrollable load, and the DGs are seen as the uncontrollable load for increasing power output.
As shown in Fig. 5b, the response order of the DSRs for decreasing power output is the discharging EVs to stop discharging, the "OFF" state TCLs to switch on, the idle EVs to recharge, the DGs to decrease power generation and then the uncontrollable DSRs. The response order for decreasing power output is shown as follows: (1) the discharging EV with lower state value will have the priority to stop discharging; (2) the "OFF" state TCL with lower state

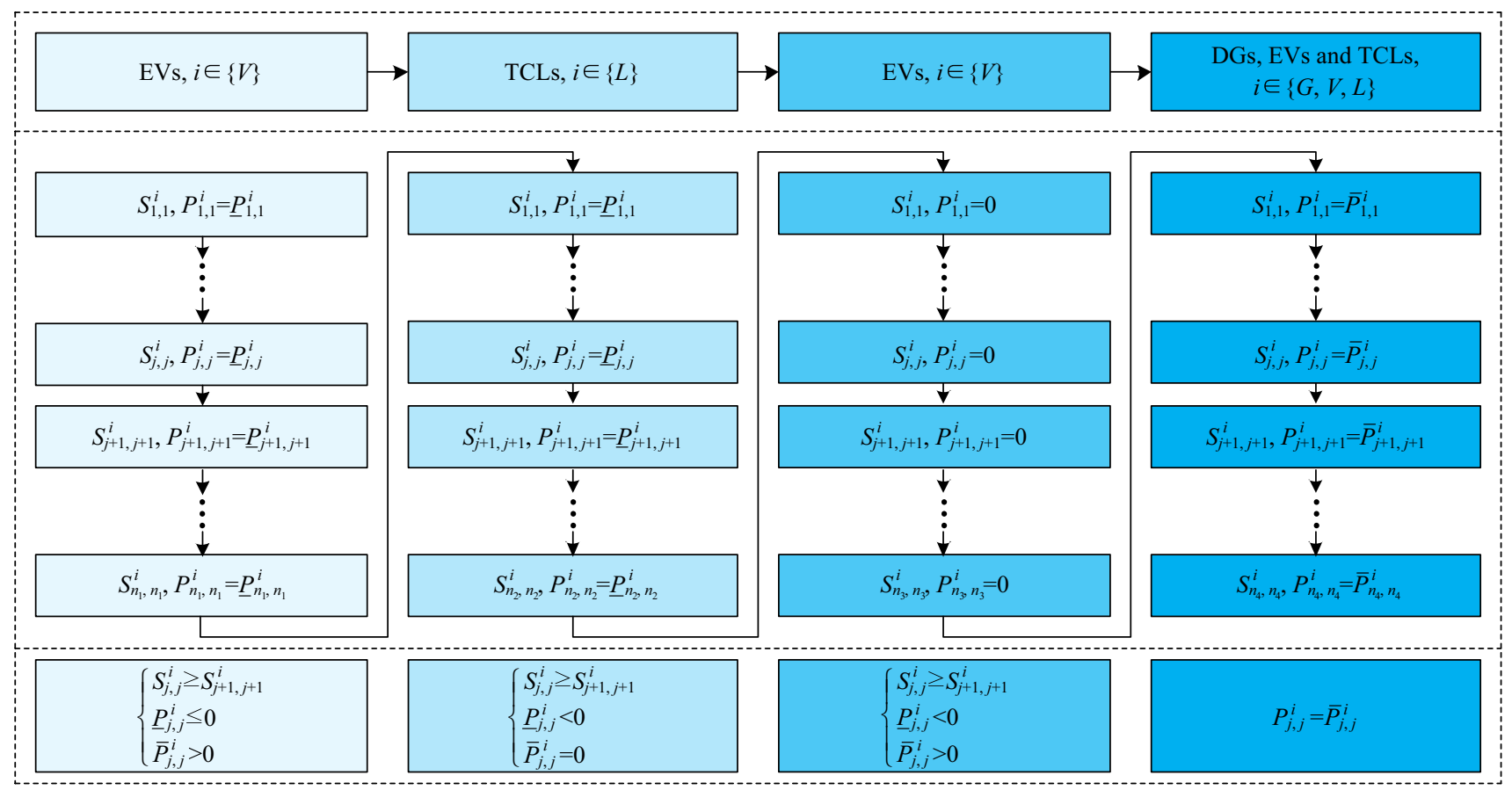

(a) Response order for increasing power output

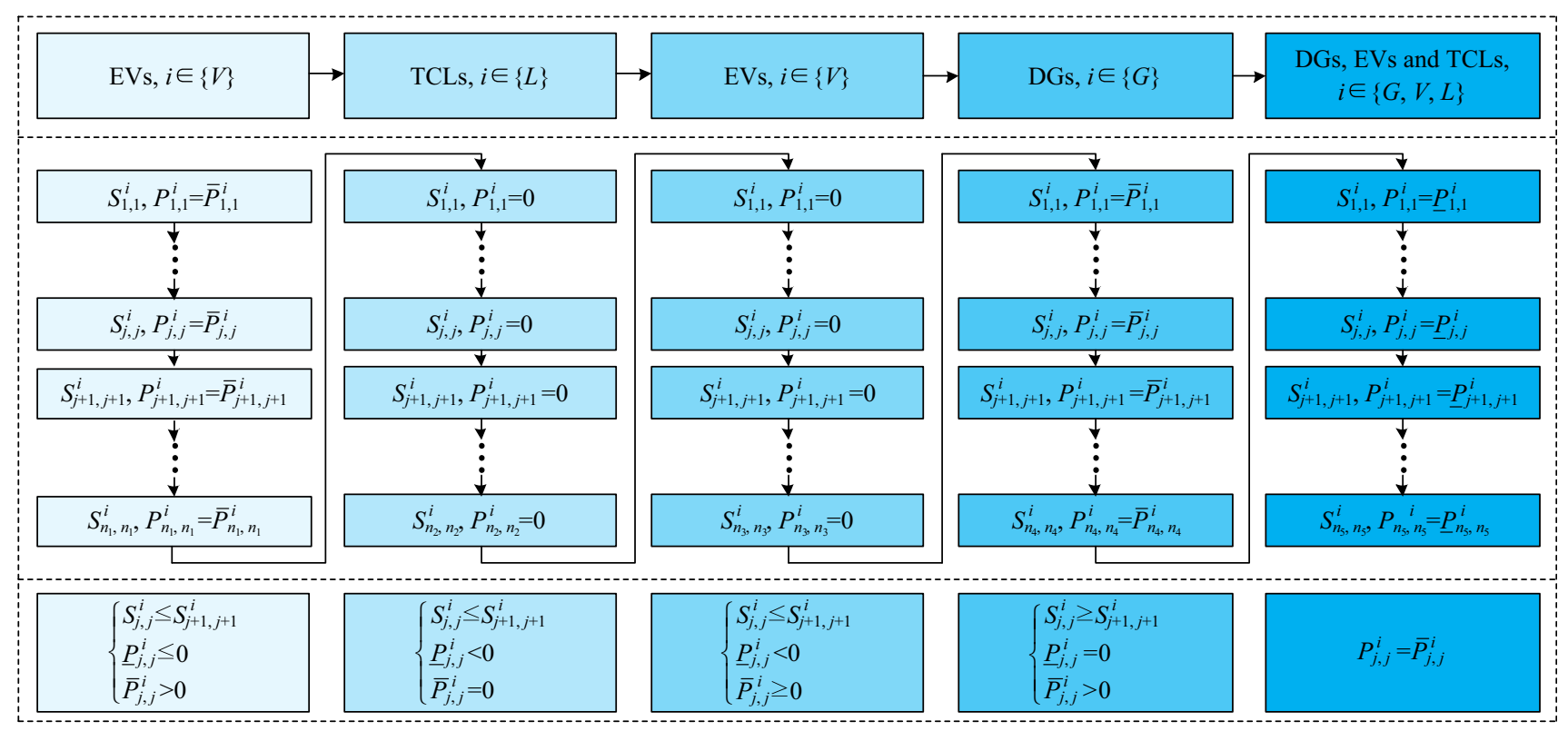

(b) Response order for decreasing power output

Fig. 5 Response order of DSRs 
value will have the priority to switch on; (3) the idle EV with lower state value will have the priority to charge; (4) the DG with higher state value will have the priority to decrease power generation; (5) the DSRs, which have reached their lower power output limitations, can be seen as the uncontrollable load.

\section{Case studies and simulation results}

In this section, a residential region introduced in [23] with households and high photovoltaic penetration is used to realize the load power smoothing control for the load power of the tie-line. The power capacity and the household number of the residential region are assumed to be the same with an IEEE 33-node distribution network with the maximum load power of $5084 \mathrm{~kW}$ introduced in [27]. To determine the number of the households, it is assumed that there are 10-40 households on each bus and each household has been installed with rooftop photovoltaic panels [28]. It is also assumed that the rooftop area varies within the range of $\left[60,100 \mathrm{~m}^{2}\right]$ and the power density is $100 \mathrm{~W} /$ $\mathrm{m}^{2}$. The daily profiles of the photovoltaic generation and the uncontrollable load are provided by UKGDS [29].

The number of EVs per household is set to be 1.86 [30]. These EVs are privately owned, and $67 \%$ of them are home-based-work EVs and 33\% of them are home-basedother EVs [31]. The traveling time distributions of the start traveling time $\left(t_{j}^{V d}\right)$ and the finish traveling time $\left(t_{j}^{V s}\right)$ are shown in Fig. 6 [32]. The rated charging and discharging power $\left(P_{j, t}^{V c}\right.$ and $P_{j, t}^{V d}$ ) of the EVs is $7 \mathrm{~kW}$ [33]. It is assumed that the EVs start charging as soon as their daily trips are finished. The other parameters, such as the battery capacity $\left(Q_{j}^{V}\right)$, the charging and discharging efficiencies $\left(\eta_{j}^{V c}\right.$ and $\left.\eta_{j}^{V d}\right)$, the minimum expected SOC $\left(S O C_{j}^{V d}\right)$, the SOC lower and upper limitations $\left(\underline{S O C}_{j}^{V}\right.$ and $\left.\overline{S O C}_{j}^{V}\right)$, are given in [33].

Each household is assumed to have its own TCL of heat pumps. The rated power consumption $\left(P_{j, t}^{L c}\right)$ of the TCL is $6 \mathrm{~kW}$ [34]. The other parameters, such as the outdoor temperature $\left(T_{j, t}^{\text {Lout }}\right)$, the limitations of the room temperature $\left(\underline{T}_{j}^{L}\right.$ and $\left.\bar{T}_{j}^{L}\right)$, the upper and lower thermostat settings $\left(\underline{T}_{j}^{L c o n}\right.$ and $\left.\bar{T}_{j}^{L c o n}\right)$, the thermal parameters $\left(R_{j}\right.$ and $\left.C_{j}\right)$, are provided by [17].

\subsection{Control effect of load curve smoothing}

Without the load curve smoothing control, the power outputs of the DGs, the EVs, the TCLs, the non-controllable load and the tie-line total load power in the residential region are shown in Fig. 7. The load curve of the TCL

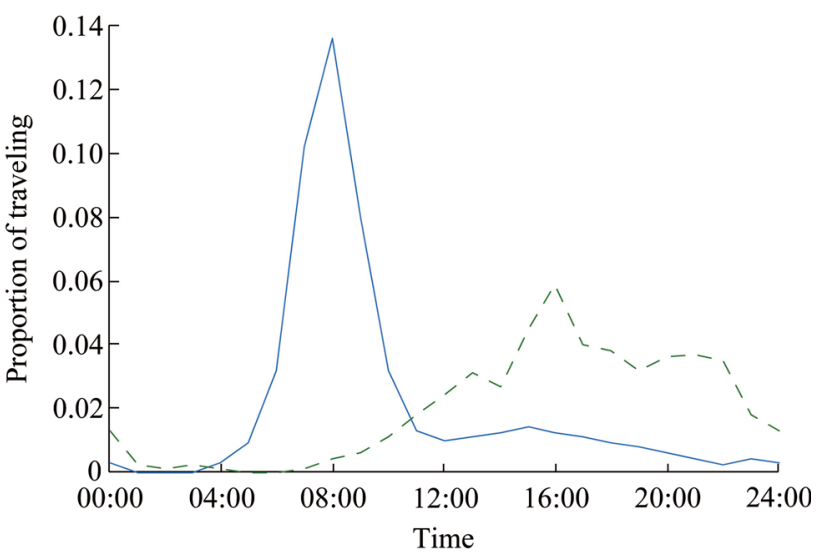

(a) Start traveling time (i.e. finish charging time)

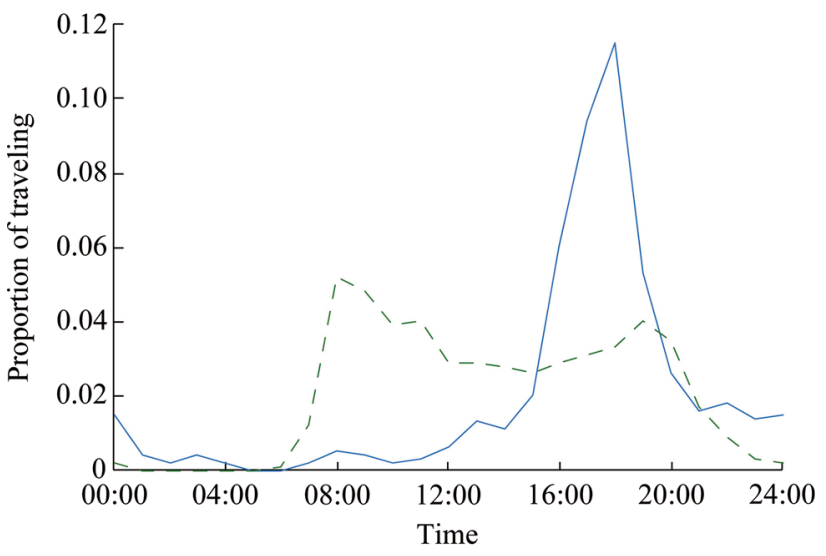

(b) Finish traveling time (i.e. start charging time)

- Home-based work EVs; - - - Home-based other EVs

Fig. 6 Traveling time distributions of EVs

power is flat, and the charging load peak of the EVs appears in 16:00-20:00. It is obvious that the power fluctuations of the total load power are primarily caused by the intermittent power outputs of the DGs during the daytime. The response capacity from the DGs, the EVs and the TCLs are used to smooth the power fluctuations of the tieline.

The limitation of the power fluctuation rate $\left(r^{T}\right)$ is assumed to be $10 \%$ per $15 \mathrm{~min}$ [11]. The total load power $\left(P_{t}^{D}\right)$ without control, the target smoothing load power $\left(P_{t}^{*}\right)$ and the actual load power with control during a day are compared in Fig. 8a. It is clear that the power fluctuations of the load are effectively smoothed. In order to further illustrate the smoothing effect of the load power in the time period with high power fluctuations, the load power without control and with control from 08:00 to 15:00 are shown in Fig. 8b. It is obvious that the actual load power exactly follows the target load power. The total responded power from all the DSRs during the day are shown in Fig. 8c.

In the existing researches of [15-23], the control signal was responded by only one type of DSR aggregator. In 


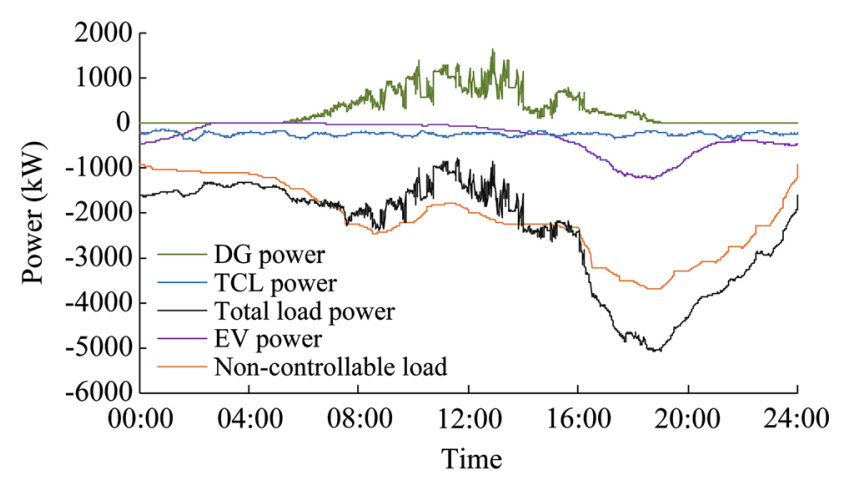

Fig. 7 Power outputs of DSRs without load curve smoothing

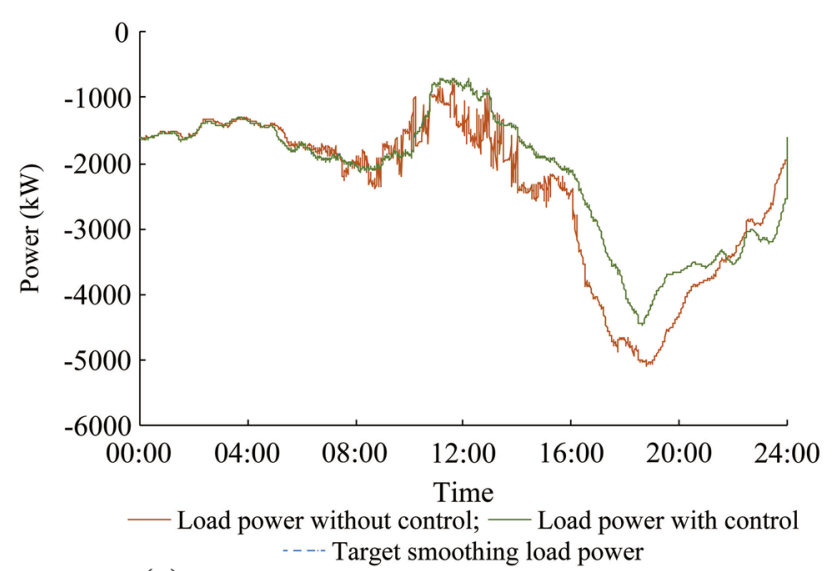

(a) Total load power without and with control during a day

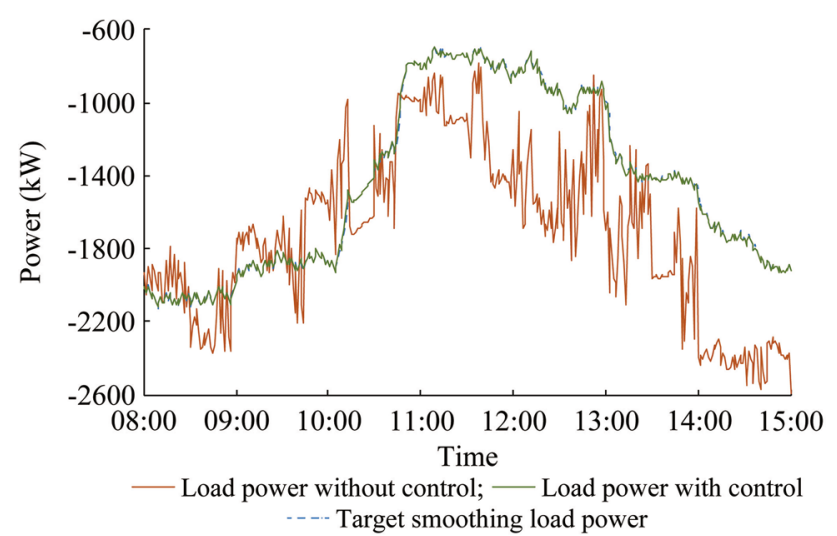

(b) Total load power without and with control during 08:00 to 15:00

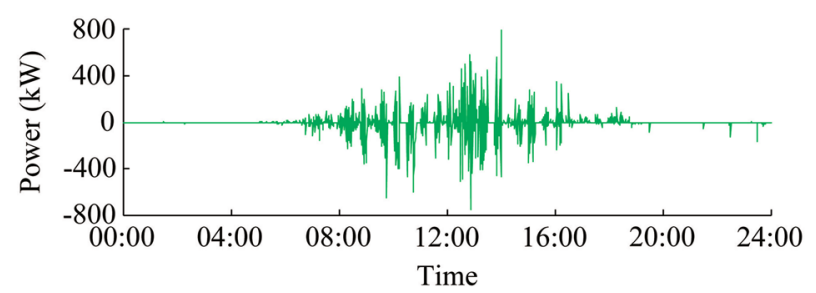

(c) Total responded power of all DSRs with control

Fig. 8 Load power without control and with control order to show the advantages of the proposed load curve smoothing control strategy, the power fluctuation rate of the load under four different control strategies is compared in Fig. 9. The four control strategies are marked with (1)-(4): (1) without any control of DSRs; (2) with the proposed control strategy based on the unified state model; (3) with the control of only the EV aggregator; (4) with the control of only the TCL aggregator. Under the proposed control strategy of (2), it is obvious that the power fluctuation rate is effectively controlled and decreased within the threshold of $10 \%$. While under the control strategies of (3) and (4), the power fluctuation rate is above the threshold of $10 \%$ during some time periods of the day. Under the control strategy of (4), the power fluctuation rate is even higher than the value without any control strategy of (1) during 12:00-13:00. This is because a large number of TCLs that are in the "OFF" state during the previous control period (12:00-13:00) have to be switched on when their lower temperature limitations are reached, which can be seen as a "secondary disturbance". And the power fluctuation becomes more serious with the sudden increase of the load power caused by the TCLs, which is further explained in Sect. 5.3.

\subsection{Function of unified state model}

In order to introduce the function of the unified state model in the smoothing control, the response order, the response capacity and the control matrix are illustrated in this section. Meanwhile, different scenarios are compared to show the advantages of the unified state model.

During the smoothing control, the response order of the DSRs is the row order of these resources in the unified state model. In order to illustrate this situation, the response order of all the DSRs during the day for increasing power output (Fig. 5a) and decreasing power output (Fig. 5b) are shown in Fig. 10. Based on the response order discussed in Sect. 4.2, for increasing the power output, the distributions of the number of EVs to stop charging, the number of TCLs to switch off, the number of EVs to discharge and the number of uncontrollable resources during a day are shown in Fig. 10a. For decreasing the power output, the distributions of the number of EVs to stop discharging, the

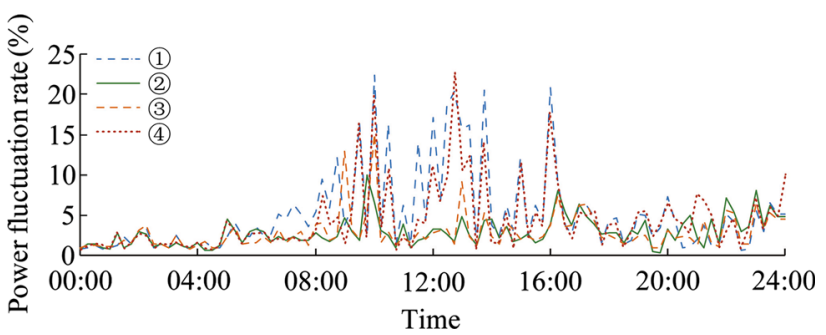

Fig. 9 Power fluctuation rate of load under different control strategies 
number of TCLs to switch on, the number of EVs to charge and the number of uncontrollable resources during a day are shown in Fig. 10b.

During the smoothing control, the response capacity of all the DSRs is obtained based on the unified state model (see (32)). The response capacity of the DSRs for increasing power output $\left(\boldsymbol{P}^{\text {up* }}\right)$ and decreasing power output $\left(\boldsymbol{P}^{\mathrm{dn} *}\right)$ is shown in Fig. 11. In Fig. 11a, the point $\left(t_{\text {inc }}\right.$, $\left.m_{\text {inc }}, P_{\text {inc }}\right)$ in the surface of the picture means the response capacity of the $1 \sim m_{i n c}$ resources for increasing power output at time $t_{i n c}$ is $P_{i n c}$ under the response order illustrated in Fig. 10a. In Fig. 11b, the point $\left(t_{d e c}, m_{d e c}, P_{d e c}\right)$ in the surface of the picture means the response capacity of the $1 \sim m_{d e c}$ resources for decreasing power output at time $t_{d e c}$ is $P_{d e c}$ under the response order illustrated in Fig. 10b.

In order to illustrate the $\boldsymbol{B}$ in the smoothing control, the values of the diagonal elements of $\boldsymbol{B}$ before control and after control at 12:30 with a response power of $-505.7 \mathrm{~kW}$ and at 13:30 with a response power of $454.4 \mathrm{~kW}$ are shown in Fig. 12.

Compared with the existing researches, the switching number of TCLs and the discharged energy of EVs are introduced to show the advantages of the unified state model for the smoothing control under the following three scenarios.

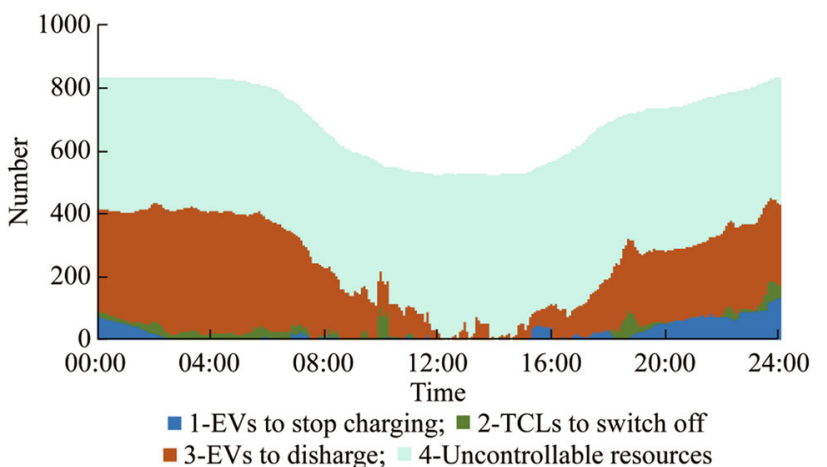

(a) Response order of DSRs for increasing power output

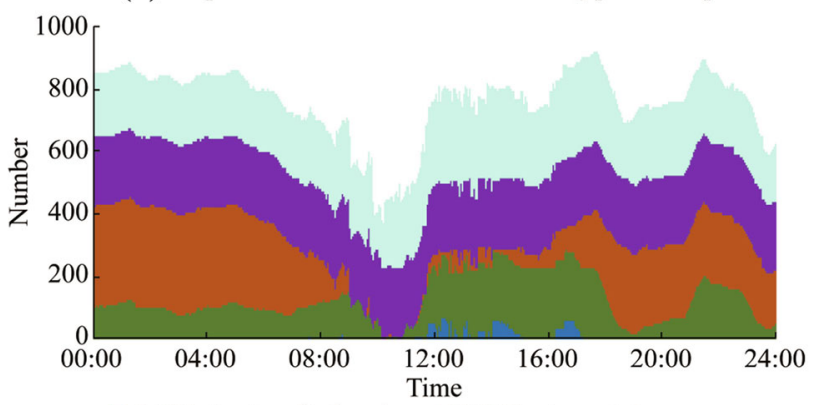

- 1-EVs to stop discharging; 1 -TCLs to switch on

- 3-EVs to charge; 4-DGs to curtail; 5-Uncontrollable resources

(b) Response order of DSRs for decreasing power output

Fig. 10 Response order of all DSRs

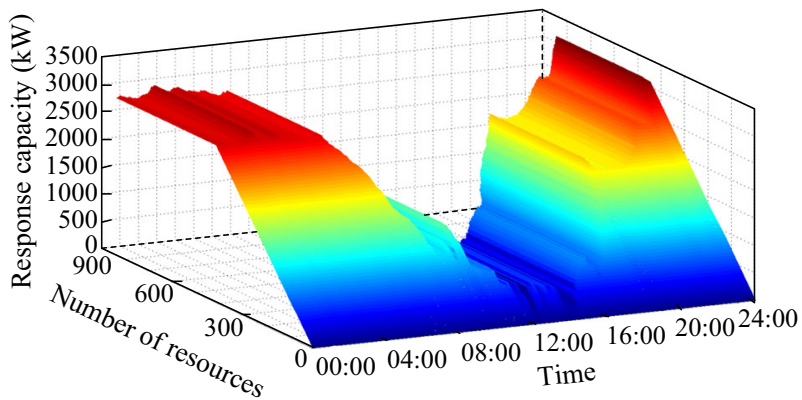

(a) Response capacity of DSRs for increasing power output

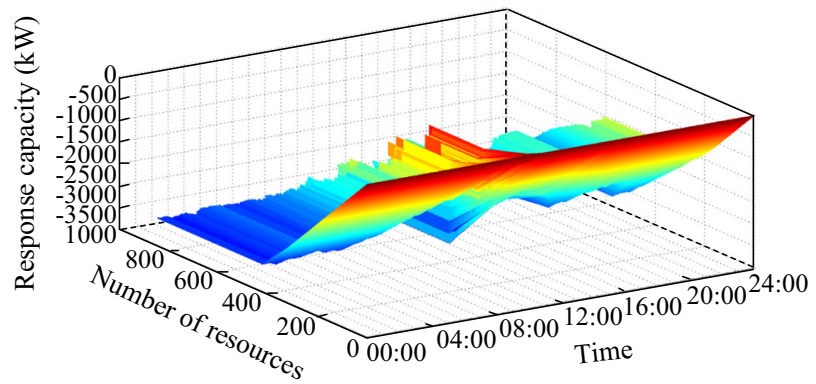

(b) Response capacity of DSRs for decreasing power output

Fig. 11 Response capacity of all DSRs

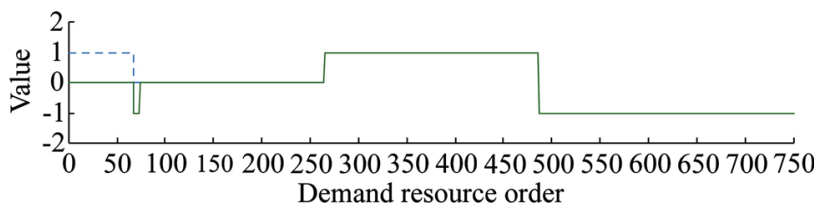

(a) Values of the diagonal elements of control matrix at 12:30

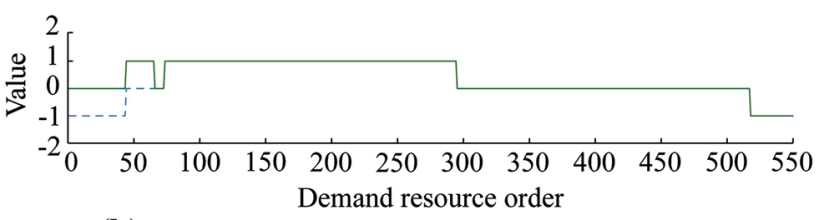

(b) Values of the diagonal elements of control matrix at 13:30 ----Before control; —— After control

Fig. 12 Power output control matrix of all DSRs

1) Scenario I: the unified state model is used for the smoothing control.

2) Scenario II: the TCL aggregator is first used for the smoothing control and the remaining unbalanced power is compensated by the EV aggregator.

3) Scenario II: the EV aggregator is first used for the smoothing control and the remaining unbalanced power is compensated by the TCL aggregator.

Under each scenario, the daily switching number of each TCL and the daily discharged energy of each EV are counted during the smoothing control, and then the average daily switching number of TCLs and the total daily discharged energy of EVs are calculated. Under the three 
scenarios, the probability density of the daily switching number is compared in Fig. 13a, where the average daily switching number of Scenario I, II, III is $21.518,30.960$, 11.275 respectively, and the probability density of the daily discharged energy is compared in Fig. 13b, where the total daily discharged energy of Scenario I, II, III is $0.998 \times 10^{3}$ $\mathrm{kWh}, 1.237 \times 10^{3} \mathrm{kWh}, 1.673 \times 10^{3} \mathrm{kWh}$ respectively.

As the TCLs are the first choice to respond to the power fluctuations in Scenario II, the average daily switching number of TCLs is much higher than the other two scenarios. As the EVs are the first choice for demand response in Scenario III, the total discharged energy is much higher than the other two scenarios. While with the proposed unified state model of Scenario I, the average switching number of TCLs is much lower than Scenario III, and the total discharged energy is lower than Scenarios II and III. With the coordinated control strategy, the unified state model is able to take advantage of different types of DSRs with different response characteristics, and thus the required discharged energy of EVs is decreased with an acceptable increase of the switching number of TCLs in the smoothing control.

\subsection{Response characteristics of DSRs}

The power outputs of the DGs without control and with control are shown in Fig. 14. It is obvious that the DG output with control exactly follows the DG output without control most of time. This occurs because in the response order for the smoothing control, the DGs are the last choice

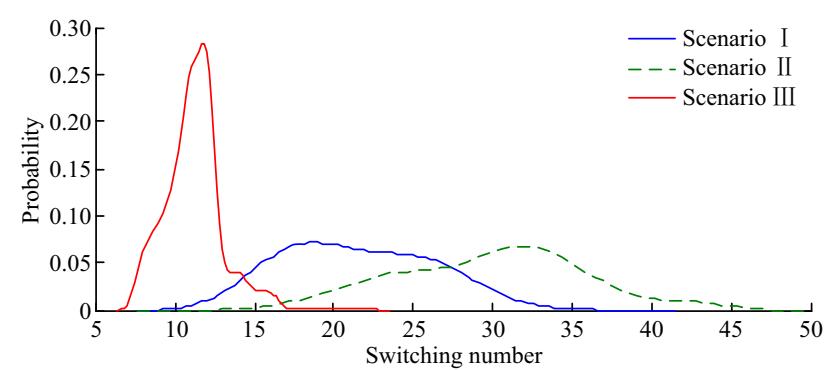

(a) Average daily switching number and probability density distribution of daily switching number of TCLs with three scenarios

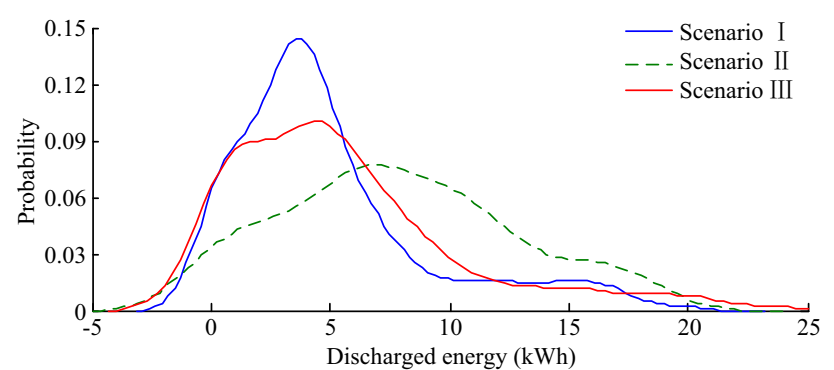

(b) Total daily discharged energy and probability density distribution of daily discharged energy of EVs with three scenarios

Fig. 13 Control effect with three scenarios for demand response to decrease their power output. Thus the power generations of the DGs are fully guaranteed during the demand response process. However, during 10:00 to 11:00, the DG output with control cannot exactly track the DG output without control. This occurs because during this period, the response capacity from the EVs and TCLs for decreasing power output is not sufficient, and the DGs have to decrease their power outputs to realize the smoothing control. And from Fig. 10b, it is clear that the number of the available EVs and TCLs for decreasing power output is quite small and the DGs have to decrease their power outputs to realize the smoothing control.

The power outputs of the EVs without control and with control are shown in Fig. 15. From 08:00 to 15:00, the power fluctuations of the EVs' output are considerable. Demand response of the EVs is realized by changing their connecting state with the power grid. To increase the power output, the charging EVs will stop charging and then even discharge to the power grid. Thus the total power of the EVs is sometimes higher than zero. The peak load of the EVs without control appears during 17:00 to 20:00, which is caused by the similar start charging time, and the peak load value is effectively decreased with the smoothing control.

The power outputs of the TCLs without control and with control are shown in Fig. 16. Before 05:00, the TCL outputs with control almost follow the power outputs without control. This occurs because the load power of the tie-line does not fluctuate seriously and only a small number of TCLs need to respond to the power fluctuations. After 05:00, the TCL outputs with control cannot follow the power outputs without control. From 12:00 to 17:00, the values of TCL outputs with control are close to zero, which means that almost all the TCLs switch off to respond to the positive power fluctuations. However, from 18:00 to 19:00, the peak load of the TCLs with control appears. This occurs because with the decreasing of the room temperature, the TCLs in the "OFF" state need to be switched on gradually.

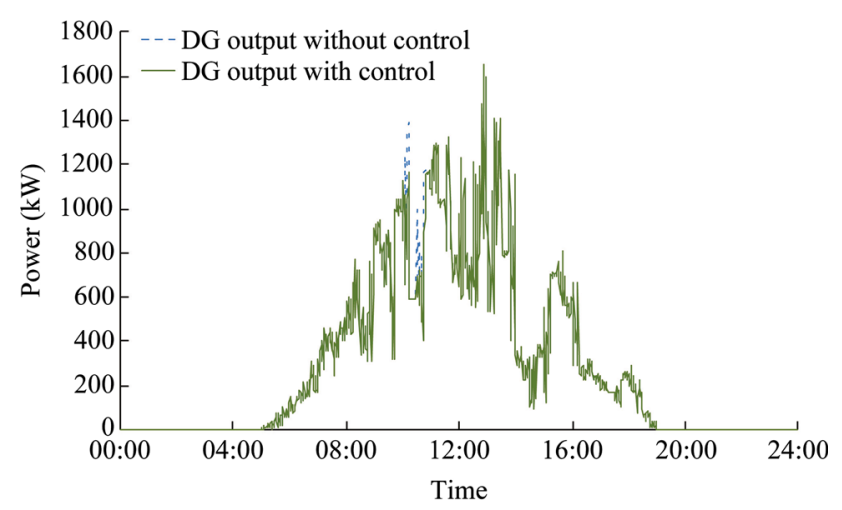

Fig. 14 Power output of DGs without control and with control 


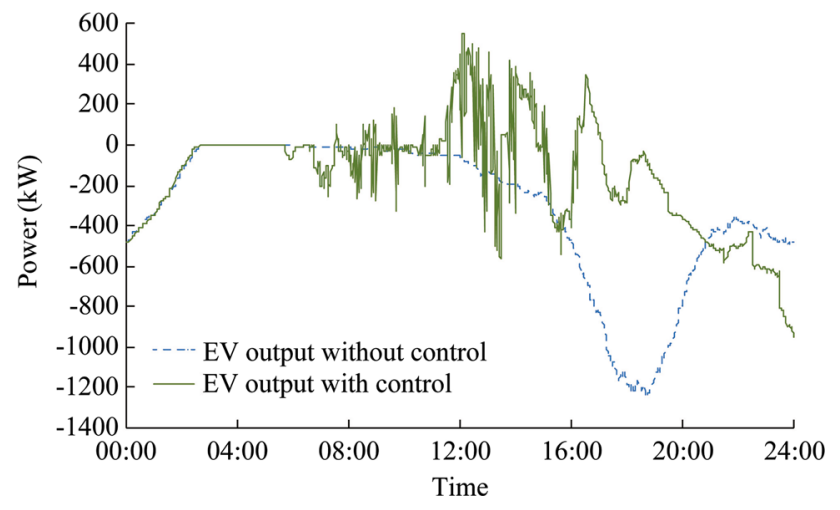

Fig. 15 Power output of EVs without control and with control

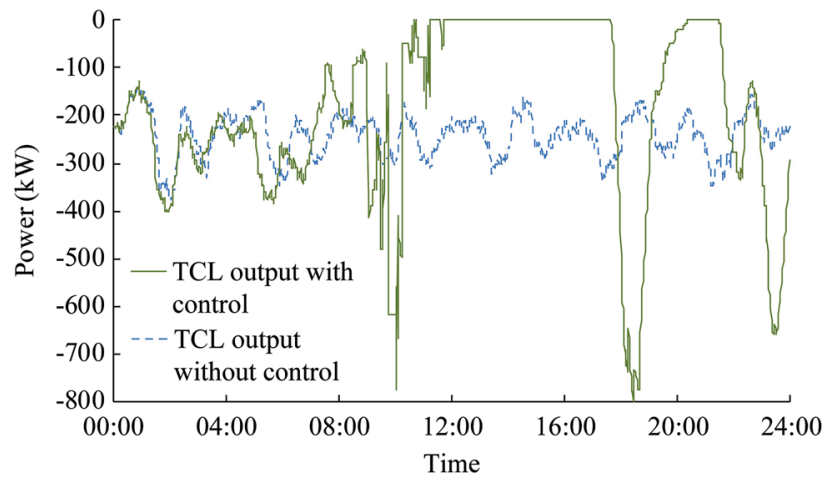

Fig. 16 Power output of TCLs without control and with control

To further introduce the response characteristics of the EVs in the load curve smoothing control, the SOCs of the EVs without control and with control are compared in Fig. 17. Each line in the figures means the SOC variations of an EV when connected to the power grid, and the SOC variations during the EV's traveling period are not illustrated. The line segment with an upward trend indicates the EV is charging power (charging state). The line segment with a downward trend indicates the EV is discharging power (discharging state). The horizontal line segment means the EV is just connected to the power grid and the power output is zero (idle state). In the load curve smoothing control, the demand response of EVs is realized by changing their connecting state ("charging $\leftrightarrow$ idle $\leftrightarrow$ discharging").

From 08:00 to 15:00, most of the EVs are traveling and there are a small number of EVs to respond to the power fluctuations. From 15:00 to 20:00, most of the EVs finish their daily travel and start to charge. The charging process is not disturbed without the load curve smoothing strategy as shown in Fig. 17a. However, the SOC variations of EVs with control are quite different as shown in Fig. 17b. From 10:00 to 11:00, all the controllable EVs are controlled to charge power to respond to the positive load power fluctuation, and the remaining unbalanced power is

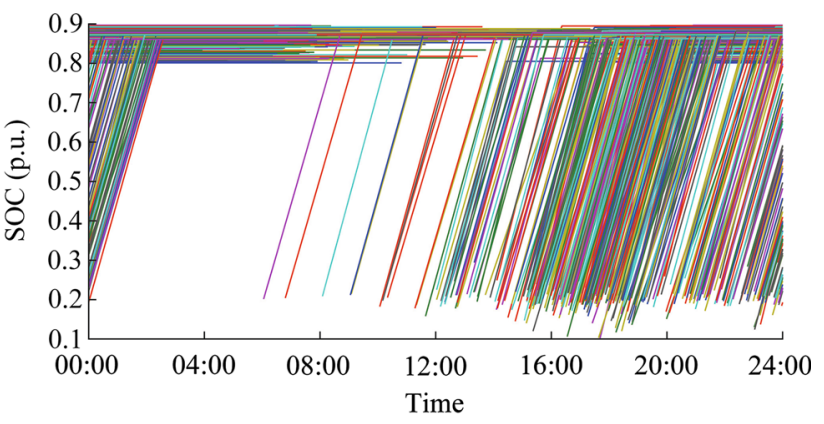

(a) Without control

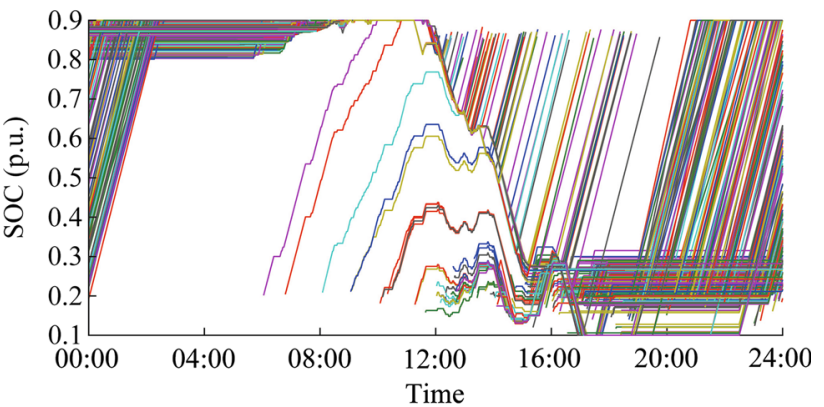

(b) With control

Fig. 17 SOC variations of EVs without control and with control

compensated by TCLs and DGs (see Figs. 14 and 15). From 13:00 to 17:00, many EVs are forced to charge power so as to satisfy the EV users' traveling demand before departure.

The room temperatures of the TCLs without control and with control are compared in Fig. 18. Each line in the figures means the room temperature variations of a TCL during the whole day. The line segment with an upward trend means the room temperature is increasing and the TCL is in the "ON" state. While the line segment with a downward trend means the room temperature is decreasing and the TCL is in the "OFF" state.

From Fig. 18a, it is obvious that the room temperature regularly varies between $19^{\circ} \mathrm{C}$ and $23^{\circ} \mathrm{C}$. From Fig. $18 \mathrm{~b}$, the room temperature with control is quite different. From 10:00 to 11:00, all the controllable TCLs are turned on to respond to the positive load power fluctuation. Considering the power output of the TCLs in Fig. 16, there are no TCLs in the "ON" state from 12:00 to 17:00, which further verifies that the response capacity is limited during this period. From 14:00 to 18:00, the TCLs are not controlled to turn on, because the response capacity from the EVs is sufficient and the TCLs do not need to respond to the power fluctuations.

Based on the unified state model, the power fluctuations of the tie-line of the residential region are effectively decreased with the load curve smoothing control. At the same time, the comfort levels of the power consumers are guaranteed during the process of controlling the power output of the DSRs. 


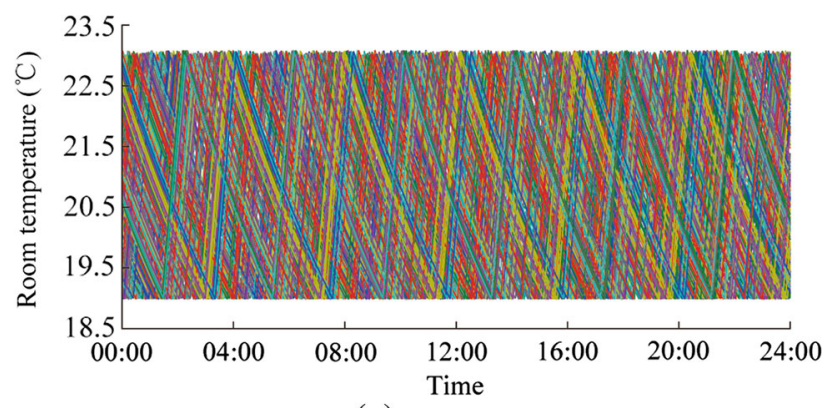

(a) Without control

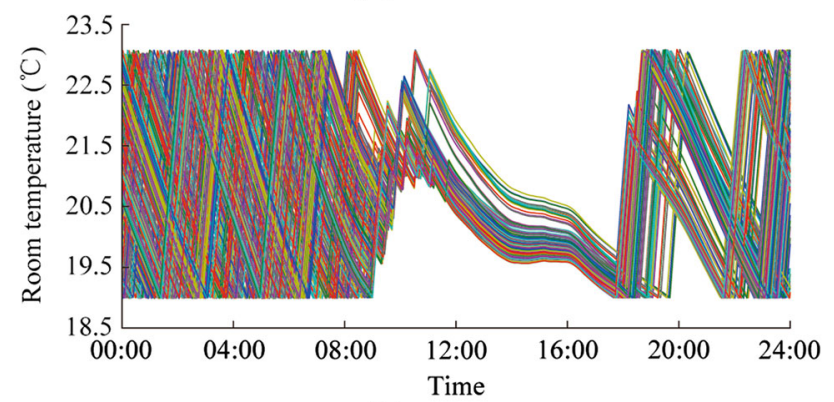

(b) With control

Fig. 18 Room temperature of TCLs without control and with control

\section{Conclusion}

This work proposed a unified state model of the DSRs, with which the response capacity is utilized for the load curve smoothing. The simulation results show that the power fluctuation rate is decreased with the proposed unified state model based load curve smoothing strategy. The conclusions are summarized as follows:

1) The smoothing control strategy based on the unified state model is able to decrease the power fluctuation rate within a preset threshold. Compared with other control strategies, the proposed control strategy takes better advantage of the response characteristics of different types of DSRs and has a better smoothing control effect without a "secondary disturbance" of the load power.

2) The unified state model is able to describe different types of DSRs with a unified mathematical expression, which gives a new solution for the DSR management in the aggregator.

3) The comparative results show that the unified state model with the coordinated control strategy can decrease the total discharged energy of the EVs with an acceptable increase of the switching number of TCLs.

4) Considering the response order of the DSRs, the power output control matrix is deduced from the unified state model to realize the real-time power output control of the DSRs. Once the required power change of the DSRs is determined, the values of the control variables in the power output control matrix are correspondingly generated.
5) The comfort levels of power consumers are considered when implementing the smoothing control with the unified state model. The SOC level of the EV is guaranteed when it starts traveling, the temperature of the room varies within an appropriate range and the renewable energy from the DGs is utilized as much as possible.

Acknowledgement This work was supported by National High Technology Research and Development Program of China (863 Program) (No. 2015AA050403), National Natural Science Foundation of China (No. 51677124, No. 51607033, No. 51607034), and Research and Demonstration on Combined Optimal Operation and Testing Technology for New Distributed Energy, Energy Storage and Active Load of State Grid Corporation of China.

Open Access This article is distributed under the terms of the Creative Commons Attribution 4.0 International License (http:// creativecommons.org/licenses/by/4.0/), which permits unrestricted use, distribution, and reproduction in any medium, provided you give appropriate credit to the original author(s) and the source, provide a link to the Creative Commons license, and indicate if changes were made.

\section{References}

[1] Yang F, Sun Q, Han QL et al (2016) Cooperative model predictive control for distributed photovoltaic power generation systems. IEEE J Emerg Sel Top Power Electron 4(2):414-420

[2] Cheng L, Chang Y, Huang R (2016) Mitigating voltage problem in distribution system with distributed solar generation using electric vehicles. IEEE Trans Sustain Energy 6(4):1475-1484

[3] Ghiani E, Pilo F (2015) Smart inverter operation in distribution networks with high penetration of photovoltaic systems. J Mod Power Syst Clean Energy 3(4):504-511

[4] Denholm P, Margolis R (2007) Evaluating the limits of solar photovoltaics (PV) in electric power systems utilizing energy storage and other enabling technologies. Energy Policy 35(9):4424-4433

[5] Zheng Y, Dong Z, Huang S et al (2015) Optimal integration of mobile battery energy storage in distribution system with renewables. J Mod Power Syst Clean Energy 3(4):589-596

[6] Boukettaya G, Krichen L (2014) A dynamic power management strategy of a grid connected hybrid generation system using wind, photovoltaic and flywheel energy storage system in residential applications. Energy 71:148-159

[7] Li K, Xu H, Ma Q et al (2014) Hierarchy control of power quality for wind-battery energy storage system. IET Power Electron 7(8):2123-2132

[8] Wang Y, Tan KT, Peng XY et al (2016) Coordinated control of distributed energy storage systems for voltage regulation in distribution networks. IEEE Trans Power Delivery 31(3):1132-1141

[9] Jayasekara NA, Masoum MS, Wolfs PJ (2016) Optimal operation of distributed energy storage systems to improve distribution network load and generation hosting capability. IEEE Trans Sustain Energy 7(1):250-261

[10] Parra D, Norman SA, Walker GS et al (2016) Optimum community energy storage system for demand load shifting. Appl Energy 174:130-143 
[11] Li X, Hui D, Lai X (2013) Battery energy storage station (BESS)-based smoothing control of photovoltaic (PV) and wind power generation fluctuations. IEEE Trans Sustain Energy 4(2):464-473

[12] Samper M, Flores D, Vargas A (2016) Investment valuation of energy storage systems in distribution networks considering distributed solar generation. IEEE Lat Am Trans 14(4):1774-1779

[13] Olek B, Wierzbowski M (2015) Local energy balancing and ancillary services in low-voltage networks with distributed generation, energy storage, and active loads. IEEE Trans Ind Electron 62(4):2499-2508

[14] Mu Y, Wu J, Jenkins N et al (2015) A spatial-temporal model for grid impact analysis of plug-in electric vehicles. Appl Energy 114:456-465

[15] Moradzadeh M, Van DVJ, Vandevelde L (2014) Optimal energy storage sizing based on wind curtailment reduction. In: Proceedings of 3rd international conference on renewable energy research and applications (ICRERA 2014), Piscataway, USA, 19-22 October 2014, pp 331-335

[16] Long C, Farrag MEA, Zhou C et al (2013) Statistical quantification of voltage violations in distribution networks penetrated by small wind turbines and battery electric vehicles. IEEE Trans Power Syst 28(3):2403-2411

[17] Lu N (2012) An evaluation of the HVAC load potential for providing load balancing service. IEEE Trans Smart Grid 3(3): $1263-1270$

[18] Luo X, Chan KW (2013) Real-time scheduling of electric vehicles charging in low-voltage residential distribution systems to minimise power losses and improve voltage profile. IET Gener Transm Distrib 8(3):516-529

[19] Deilami S, Masoum AS, Moses PS et al (2011) Real-time coordination of plug-in electric vehicle charging in smart grids to minimize power losses and improve voltage profile. IEEE Trans Smart Grid 2(3):456-467

[20] Shao S, Pipattanasomporn M, Rahman S (2011) Demand response as a load shaping tool in an intelligent grid with electric vehicles. IEEE Trans Smart Grid 2(4):624-631

[21] Tehrani NH, Wang P (2015) Probabilistic estimation of plug-in electric vehicles charging load profile. Electr Power Syst Res 124:133-143

[22] Mammoli A, Barsun H, Burnett R et al (2012) Using high-speed demand response of building HVAC systems to smooth clouddriven intermittency of distributed solar photovoltaic generation. In: 2012 IEEE PES transmission and distribution conference and exposition, Orlando, USA, 7-10 May 2012, 10 pp

[23] Wang D, Ge S, Jia $\mathrm{H}$ et al (2014) A demand response and battery storage coordination algorithm for providing microgrid tie-line smoothing services. IEEE Trans Sustain Energy $5(2): 476-486$

[24] Guo B, Yang J, Qu B et al (2016) Coordination frequency control strategy for EVs and thermostatically controlled appliances in the power system. Proc CSU-EPSA 28(12):13-18

[25] Papadaskalopoulos D, Strbac G, Mancarella P et al (2013) Decentralized participation of flexible demand in electricity markets-Part II: application with electric vehicles and heat pump systems. IEEE Trans Power Syst 28(4):3667-3674

[26] Ma Y, Houghton T, Cruden A et al (2012) Modeling the benefits of vehicle-to-grid technology to a power system. IEEE Trans Power Syst 27(2):1012-1020

[27] Rao RS, Ravindra K, Satish K et al (2015) Power loss minimization in distribution system using network reconfiguration in the presence of distributed generation. IEEE Trans Power Syst 28(1):317-325

[28] Yao E, Samadi P, Wong VWS et al (2016) Residential demand side management under high penetration of rooftop photovoltaic units. IEEE Trans Smart Grid 7(3):1597-1608
[29] University of Strathclyde (2006) United Kingdom generic distribution system (UKGDS). http://monaco.eee.strach.ac.uk/ ukgds/. Accessed 14 March 2016

[30] Yao W, Chung CY, Wen F et al (2016) Scenario-based comprehensive expansion planning for distribution systems considering integration of plug-in electric vehicles. IEEE Trans Power Syst 31(1):317-328

[31] Wang M, Mu Y, Jia $\mathrm{H}$ et al (2015) A preventive control strategy for static voltage stability based on an efficient power plant model of electric vehicles. J Mod Power Syst Clean Energy 3(1):103-113

[32] Wang M, Mu Y, Jia H et al (2017) Active power regulation for large-scale wind farms through an efficient power plant model of electric vehicles. Appl Energy 185:1673-1683

[33] Yu T, Yao X, Wang M et al (2016) A reactive power evaluation model for EV chargers considering travelling behaviors. In: Proceedings of 5th international conference on electric utility deregulation and restructuring and power technologies (DRPT), Changsha, China, 26-29 November 2015, 9 pp

[34] Wang D, Fan M, Jia H (2014) User comfort constraint demand response for residential thermostatically-controlled loads and efficient power plant modeling. Proc CSEE 34(13):2071-2077

Mingshen WANG received his B.S. and M.S. degrees in electrical engineering from Tianjin University, China, in 2013 and 2016 respectively. He is currently pursuing the Ph.D. degree in the Electrical Engineering Department, Tianjin University. His research interests include demand response and renewable energy integration.

Yunfei MU received his B.S., M.S., and Ph.D. degrees in electrical engineering from Tianjin University, China, in 2007, 2009, and 2012 respectively. He is an associated professor of Tianjin University. His research interests include power system stability analysis and control, renewable energy integration and electrical vehicles.

Tao JIANG received his B.S. and M.S. degrees in electrical engineering from Northeast Electric Power University, Jilin, China, in 2006 and 2011, respectively, and the Ph.D. degree in electrical engineering from Tianjin University, Tianjin, China, in 2015. He is currently an associate professor with the Department of Electrical Engineering, Northeast Electric Power University, Jilin, China. His research interests include power system stability analysis and control, renewable energy integration, demand response, and smart grid.

Hongjie JIA received his B.S., M.S., and Ph.D. degrees in electrical engineering from Tianjin University, China, in 1996, 1998, and 2001 respectively. He is a professor of Tianjin University. His research interests include power stability analysis and control, distribution network planning and automation, and smart grids.

Xue LI received the Ph.D. degree from the University of Tennessee (UT), Knoxville in 2015. She is presently an associated professor at Northeast Electric Power University, Jilin, China. Her research interests include parallel computing in power system analysis and power-aware computations.

Kai HOU received his Ph.D. degree in electrical engineering from Tianjin University, Tianjin, China in 2016. He is currently a lecturer at Tianjin University. His research interests include reliability and risk assessments of power system, integrated energy system, and smart grid.

Tong WANG is currently a senior engineer at NARI Solar Energy Technologies Company Limited, Nanjing, China. His research interests include smart grid and microgrid. 